\title{
Reproduction regulates Drosophila nutrient intake through independent effects of egg production and sex peptide: Implications for aging
}

\author{
Elizabeth Bowman ${ }^{\mathrm{a}, \mathrm{b}}$ and Marc Tatar ${ }^{\mathrm{a}, *}$ \\ ${ }^{a}$ Department of Ecology and Evolutionary Biology, Brown University, RI, USA \\ ${ }^{\mathrm{b}}$ Department of Epidemiology, Marion County (Indiana) Public Health Department, IN, USA
}

\begin{abstract}
.
BACKGROUND: The ratio of protein to carbohydrate $(\mathrm{P}: \mathrm{C})$ consumed influences reproduction and lifespan, outcomes that are often maximized by different P:C intake.

OBJECTIVE: Determine if reproduction in female Drosophila drives elevated P:C intake. Distinguish whether such a preference is driven by egg production or from male-derived sex peptides in seminal fluid.

METHODS: Intake of protein and carbohydrate was measured in a diet-choice assay. Macronutrient intake was calculated for mated and unmated fertile females, mated and unmated sterile females, and both types of female when mated to wildtype males and to males lacking sex peptide.

RESULTS: Mated females have high P:C intake relative to unmated females and mated, sterile females. Fertile females mated to wildtype males and to males lacking sex peptide have high P:C intake, but sterile females have similar, low P:C intake when unmated and when mated to males lacking sex peptide.

CONCLUSIONS: The metabolic demands of egg production and sex peptides are individually sufficient to drive elevated P:C intake in adult female Drosophila. Reproductive state can thus modulate how animals consume macronutrients, which in turn can impact their health and aging.
\end{abstract}

Keywords: Nutrients, reproduction, sex peptide, Drosophila, aging

\section{Introduction}

Egg production and adult survival are optimized at different dietary intake ratios of protein to carbohydrate (P:C) in Drosophila [1-3]. These differences may help explain trade-offs between reproduction and aging seen in many animals [4-7]. Survival of adult Drosophila is greatest with low P:C diet while

\footnotetext{
*Corresponding author: Marc Tatar, Professor of Biology, Department Ecology and Evolutionary Biology, Brown University, Providence, RI 02912, USA. Tel.: +1 401863 3455; Fax: +1 401863 2166; E-mail: marc_tatar@brown.edu.
}

egg production rate is greatest at high $\mathrm{P}: \mathrm{C}$. When given a dietary choice, Drosophila females select an intermediate P:C that maximizes lifetime reproductive success through the product of age-specific survival and age-specific fecundity [1]. Although the mechanism for how low $\mathrm{P}: \mathrm{C}$ favors survival is unknown, high $\mathrm{P}: \mathrm{C}$ in adult diet is required to support egg production in Drosophila because females are income breeders [8-10]: eggs are built with amino acids acquired from yeast ingested by adults, not from protein carried over from the larval phase. Under these conditions, egg production demand could drive 
Drosophila females to select relatively high P:C diets. Here we explicitly study how reproduction affects diet choice by measuring the P:C intake of females that were manipulated to differ in egg production and mating.

We find that mated fertile females consumed high P:C diet compared to diet consumed by sterile females. Egg production alone does not drive nutrient selection, because P:C intake was also elevated in mated sterile females relative to unmated sterile females, consistent with previous work where mated females prefer yeast diet after a bout of food deprivation whereas unmated females do not $[11,12]$. Ribeiro and Dickson [11] further reported that female sex peptide receptor mutants did not prefer yeast after deprivation even if they were mated. Likewise, wildtype females showed weak preference for yeast if they were mated with males lacking sex peptide $\left(\mathrm{SP}^{0}\right)$ [11]. Earlier work on mating costs of reproduction in Drosophila also documented robust survival in females mated to males lacking accessory gland products or sex peptide compared to females mated to wildtype males $[13,14]$. Here we find that P:C intake was similar among wildtype females mated to wildtype and $\mathrm{SP}^{0}$ males, while these intakes were both greater than intake by virgin females. However, $\mathrm{P}: \mathrm{C}$ intake was greatly reduced when females lacking germline stem cells were mated to $\mathrm{SP}^{0}$ males. Our data suggest that egg production and sex peptide transferred from males are independently sufficient to elevate P:C intake in Drosophila females. Sex peptide may thus induce a nutrient feeding behavior that anticipates a physiological demand. Sex peptides and egg production demand will contribute to aging costs of reproduction by shifting P:C intake toward a ratio favoring reduced survival, all the while increasing fitness.

\section{Materials and methods}

Nutrient intake was measured from adult female Drosophila in a two-choice CAFE assay, following the protocol of Lee et al. [1]. Assays were conducted in 8 dram glass Drosophila culture vials containing a bottom layer of $1.5 \%$ agar media and stoppered by a foam bung with two apertures made from disposable pipette tips. Each aperture contained a snugly fit $5-\mu 1$ microcapillary tube (Drummond Microcaps) filled with either yeast solution or sugar solution. The capillary tubes extended into the glass vials where females could freely feed from either solution. To minimize effects by evaporation, all assay were conducted in a chamber with relative humidity at $60 \%$, and control vials lacking females were used to estimate fluid loss from capillary tubes independent of feeding. One or two adult female Drosophila flies of the same mating condition and genotype were housed together in each vial to measure their nutrient consumption. A single fly was used when food measurement was collected once every two days; two flies were used when food intake was measured every day. In trails where mating frequency was manipulated, nutrient intake was measured from 16 vials that held two adult females in the two-choice CAFE assay.

To initiate a trial females were collected as virgins after eclosion from rearing bottles where development was completed upon standard laboratory diet (yeast/agar/sugar/cornmeal) at controlled density, $25^{\circ} \mathrm{C}, 40 \% \mathrm{RH}, 12 \mathrm{~h}$ light: $12 \mathrm{~h}$ dark. Females were subsequently housed in fresh vials with laboratory media and with five males of appropriate genotype (mating), or alone (unmated). After 24 hours females were individually transferred to CAFE vials where they were maintained for two weeks. Depending on the particular study, fluid consumed from each food tube within each vial was measured daily or every other day. In the study where females were re-mated, females were transferred to an empty vial without (control) or with a male (re-mated) for 4 hours on day seven, and then returned to their respective CAFE vials for the remaining assay period (7 days). In the study where females were either virgin, mated to wild type males, or mated to sex peptide mutant males, the assay period was 7 days in total, with two female flies housed in each vial and with 16 vials per mating condition.

Protein and carbohydrate intake during each census interval was calculated from the measured consumption of the yeast $(90 \mathrm{~g} / \mathrm{L})$ and sugar solutions $(180 \mathrm{~g} / \mathrm{L})$. Yeast solution consisted of fine autolyzed yeast (MP Biomedicals, \#103304) in sterile, distilled water with $0.01 \%$ phosphoric acid and $0.1 \%$ propionic acid as antibiotics. This yeast contained $45 \%$ protein and $24 \%$ carbohydrates as glucose equivalents (the remainder contained fiber, water and fatty acids). Linear measures of solution consumed from each capillary tube (minus measured loss in the noanimal control vials) were converted to volumes, then to total protein and carbohydrate consumed by each female, each census interval. Mean consumption across all females in a treatment group was calculated each interval and plotted as a time dependent cumulative regression to estimate linear 
(or quadratic) functions where slope estimates the $\mathrm{P}: \mathrm{C}$ intake selected by females of each experimental group. Goodness of linear fit was compared to a quadratic model, and differences in slope (P:C intake) were tested by ANCOVA.

We compared fertile females to females made sterile by the dominant OvoD mutation [15] or by lacking germline stem cells ('Bam' females) [16]. Sterile OvoD mutants have active germline stem cells but arrest egg development and produce no eggs. Bam females express transgene bag-of-marbles (nosGAL4:VP16 > UASp-bam) in germline stem cells, which causes terminal stem cell differentiation and depletes self-renewing egg progenitors.

\section{Results}

In our first trial, females of all genotypes were mated to wildtype males for 24 hours post eclosion ('once-mated') and subsequently monitored for 14 days. Mated, fertile females (wildtype) consumed protein to carbohydrate $(\mathrm{P}: \mathrm{C})$ in a ratio of $1: 14$ (Fig. 1). Sterile Bam females also produced a linear diet intake function with a significantly lower slope with P:C of 1:17 $(p<0.006)$. Sterile OvoD females consumed diet with P:C of 1:16 based on estimates using a linear function. This value was not statistically distinct from Bam or wildtype females but comparisons are ambiguous because the intake

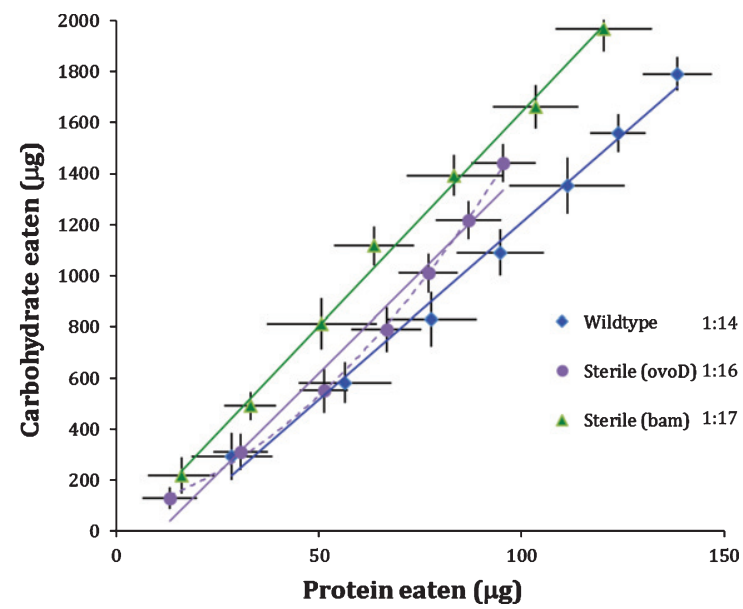

Fig. 1. Wildtype (fertile) and sterile (OvoD, Bam) females. Mean cumulative protein and carbohydrate intake consumed by females with daily point estimate standard deviation, plotted across two weeks in CAFE assay. Cumulative intake trajectories with 2-day census intervals, and best-fit linear (solid line) or quadratic (dash line) functions. P:C ratios at legend were estimated from linear regression. function of OvoD females was significantly concave $(p<0.0005)$. In general, however, mating and subsequent egg production is associated with higher protein to carbohydrate intake.

Egg production itself may not induce high P:C intake because sterile, mated OvoD females are reported to prefer yeast diet following food deprivation [11]. Furthermore, this yeast preference declines over the course of days in fertile females [11], consistent with the concave $\mathrm{P}: \mathrm{C}$ intake function we observe for once-mated OvoD females (Fig. 1). P:C preference may be transiently elevated by mating, rather than by egg production. To explore this idea, we compared $\mathrm{P}: \mathrm{C}$ functions of virgin females to females mated once and mated twice (once after eclosion and then again at day 7). The analysis was conducted with wildtype and $\mathrm{OvoD}$ females, measuring protein and carbohydrate intake daily for 14 days.

Unmated wildtype and OvoD females have linear intake functions with relatively low $\mathrm{P}: \mathrm{C}$ ratio (Wildtype 1:14; OvoD 1:20) (Fig. 2). Once-mated females of both genotypes generated significantly concave
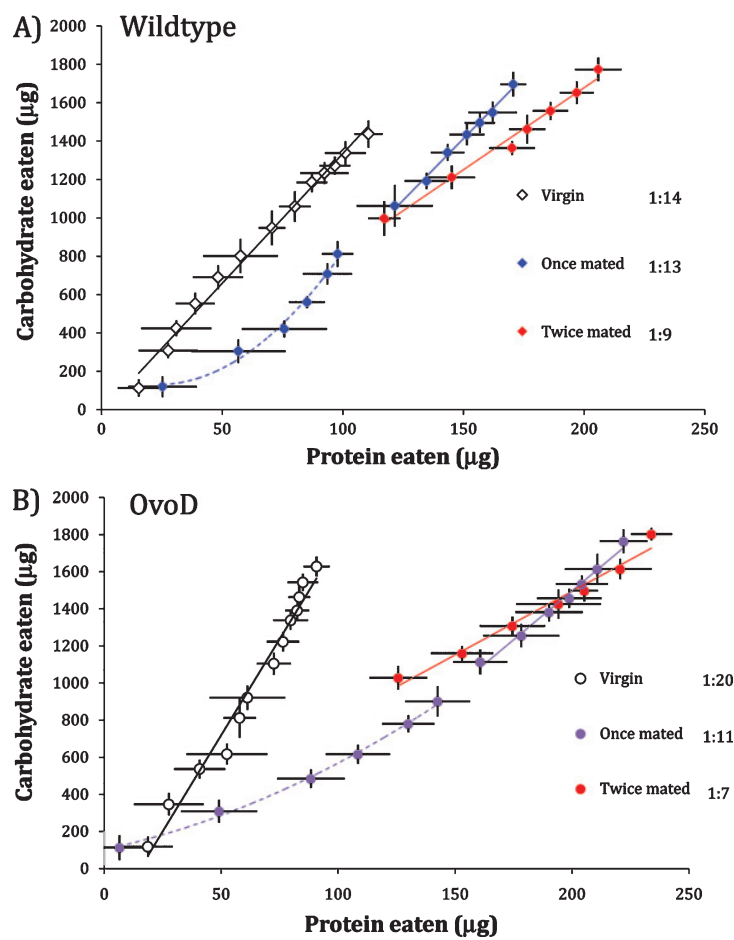

Fig. 2. Wildtype fertile females (A) and sterile OvoD females (B) when unmated (virgin), once-mated (at eclosion), or twice mated (at eclosion and remated at day 7). Cumulative intake trajectories with daily point estimate standard deviation, using daily census intervals, and best fit linear (solid line) or quadratic (dash line) functions, with $\mathrm{P}: \mathrm{C}$ ratios (at legend) estimated from linear regression. 
consumption trajectories across the six days after first mating (Fig. 2 dashed lines), indicating they initially selected high P:C diet that shifted to lower P:C. At day 7 , a subset of females was re-mated and intake functions were plotted going forward (solid lines, Fig. 2). In wildtype and OvoD females alike, remated females consumed significantly greater P:C diet than their age-matched, once mated counterparts (wildtype once mated 1:13 versus twice mated $1: 9, p<0.0004$; OvoD once mated 1:11 versus twice mated 1:7, $p<0.001)$. Because these patterns hold in both fertile (wildtype) and sterile (OvoD) females, mating appears to transiently increase P:C intake independent of egg production.

Males transfer sex peptides (SP) during copulation to affect female post mating receptivity, JH production, and egg laying rate $[17,18]$. Mutant $\mathrm{SP}^{0}$ males copulate and fertilize eggs but do not transfer the sex peptide Acp70A. Since mating affects female P:C intake independent of egg production (Fig. 2), we tested whether sex peptides were responsible for this affect, as explored by Ribeiro [11] in the context of feeding after a period of yeast deprivation. P:C intake was measured across 14 days (measured every two days) from wildtype, OvoD and Bam females when unmated (virgin) or once mated to wildtype $\left(\mathrm{SP}^{+}\right)$ or $\mathrm{SP}^{0}$ males (Fig. 3). Although P:C intakes in many of these trials were overall lower than previous estimates, the differences among treatments nonetheless revealed consistent patterns.

Among wildtype females, mating by both male genotypes strongly increased protein intake relative to virgin females $(p<0.0001)$ (Fig. 3A). These data, however, do not distinguish between drive for protein choice from egg production and potential effects of sex peptide because both male genotypes stimulated similar levels of oviposition (Fig. 4).

Analysis of sterile Bam females helps resolve the issue (Fig. 3C). Germline stem cell knock out females (Bam) mated with $\mathrm{SP}^{+}$males (control males) have elevated P:C intake (1:12) compared to unmated Bam females $(1: 27)(p<0.0001)$, but Bam females mated with $\mathrm{SP}^{0}$ males (mutant males) have $\mathrm{P}: \mathrm{C}$ intake (1:34) similar to (or less than) that of unmated females $(p<0.001)$. Thus, sex peptide is necessary and sufficient to induce high P:C intake among females lacking germline stem cells.

Sterile OvoD females produce an intermediate case (Fig. 3B). Mating to $\mathrm{SP}^{+}$males (control males) increases $\mathrm{P}: \mathrm{C}$ intake $(1: 12)$ relative to virgin females $(1: 41)(p<0.0001)$, reinforcing that mating independent of egg production favors protein intake.
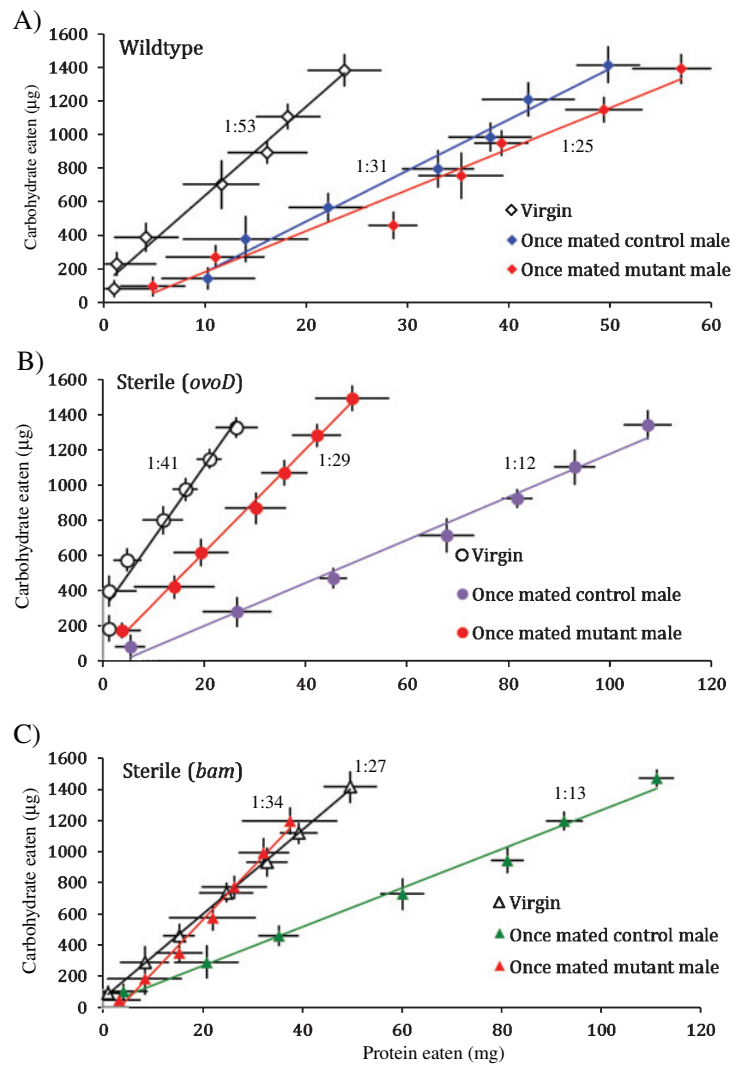

Fig. 3. Wildtype fertile females (A), sterile OvoD females (B), sterile Bam females when unmated (virgin), or once-mated (at eclosion) to wildtype males (control) or to males lacking sex peptide $\mathrm{SP}^{0}$ (mutant) (C). Cumulative intake trajectories with daily point estimate standard deviation, using 2-day census intervals, and best-fit linear function, with $\mathrm{P}: \mathrm{C}$ ratios (at legend) estimated from linear regression.

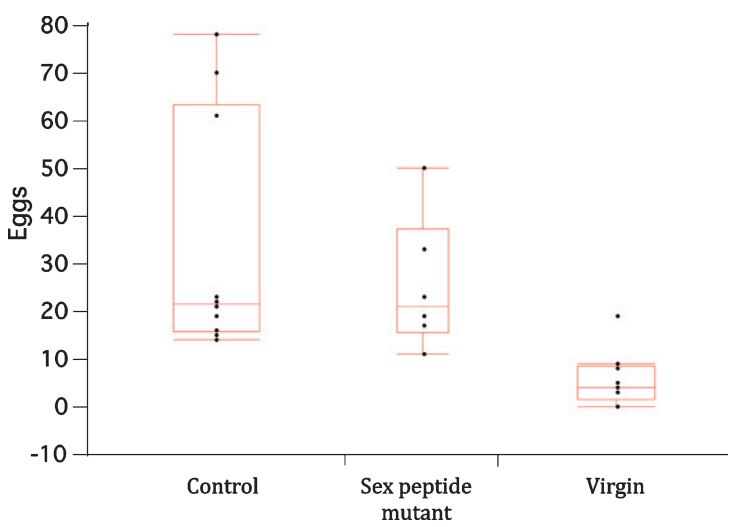

Fig. 4. Box-whisker plots for eggs laid in CAFE vials by wildtype females once mated to wildtype males (control) or to males lacking sex peptide (sex peptide mutant), or when unmated (virgin). Points represent total number of deposited eggs in the first three days of the CAFE trial. Means among the mated females did not significantly differ, while means of both mated female groups differed from that of unmated females (Dunnett's multiple comparison with $\alpha=0.05$ ). 
But OvoD females mated to $\mathrm{SP}^{0}$ males (mutant males) showed less P:C intake (1:29) than when mated to $\mathrm{SP}^{+}$males, although not as low as virgin females (both contrasts $p<0.0001$ ). Since these females produce early stage oocytes but no mature eggs, their intermediate P:C intake may be attributed to mating signals independent of sex peptide that interact with intact germline.

\section{Discussion}

Egg production and sex peptides are each sufficient to stimulate high P:C intake. Mated, fertile females have high P:C intake relative to unmated females and to sterile females, and this holds regardless of male sex peptide genotype. Egg production demand appears to be sufficient to drive female protein preference. Analysis of germline knockout Bam females suggests, however, that sex peptides are also sufficient to drive diet preference, as reported by Ribeiro [11]. Bam females when unmated and when mated to $\mathrm{SP}^{0}$ males have nearly identical, low P:C intake while Bam females mated to control males $\left(\mathrm{SP}^{+}\right)$have high $\mathrm{P}: \mathrm{C}$ intake even though they produce no eggs. Importantly, the similarity between unmated and $\mathrm{SP}^{0}$ mated Bam females suggests there are no mating-related stimuli for female diet preference besides sex peptide. Male sex peptide is sufficient to drive protein preference, but egg production is also sufficient to drive protein preference because fertile females mated to $\mathrm{SP}^{0}$ males are fecund and have high $\mathrm{P}: \mathrm{C}$ intake.

Metabolic demands of egg production could drive feeding behavior through hormones produced in the fat body, an insect tissue with adipose and liverlike function [19, 20]. Drosophila fat body produces yolk protein from amino acids acquired by adult feeding [21-23]. Yolk proteins are subsequently transported and provisioned to developing egg chambers. In principle, the metabolic demands of egg production within the fat body may affect food preference through adipokine signaling. Drosophila fat body produces leptin-like UPD2, and this induces Drosophila insulin-like peptide 2 (DILP2) from brain medial secretory neurons when adults are fed high carbohydrate diets [24]. In contrast, fat body produces DILP6 in response to dietary protein deprivation, and this response represses DILP2 secretion [25]. Both UPD2 and DILP6 are regulated in part by the dFOXO transcription factor, which itself is modulated by fly insulin-like peptides [26, 27]. Currently it is unknown if yolk protein metabolism affects adipokines, or how such hormones impact CNS regulation of feeding behavior.

Drosophila males deliver sex peptides during copulation. These sex peptides induce female mating refraction, increase the rate of egg production and stimulate synthesis of juvenile hormone $(\mathrm{JH})$, a farnesol derived terpenoid produced in the corpora allata [17, 18, 28]. Ribeiro [11] reported how sex peptides increased dietary protein preference in wildtype females following a short period of protein deprivation. Our analysis of sex peptides included wildtype and sterile females, and confirmed that sex peptide is sufficient to affect female feeding. Ribeiro also studied sex peptide receptor mutant females, including mutants where the receptor was reduced in specific neurons. These adults suggested that sex peptide is necessary to induce yeast preference after food deprivation, and the work placed the site of action in the CNS.

We note that sex peptide may as yet affect protein preference through signals associated with egg production demand. Sex peptide induces juvenile hormone, and $\mathrm{JH}$ stimulates yolk protein production in the fat body. Thus, JH induced in otherwise sterile females might still stimulate fat body metabolism and associated adipokines. In this case sex peptide receptor neurons must function upstream of the corpora allata to control JH synthesis. Such an endocrine network could help explain why both egg production and sex peptide are each sufficient to control P:C intake in females: sterility will blunt metabolic demand from the fat body (even in the face of $\mathrm{JH}$ ) and repress adipokines, and loss of sex peptide inhibits CNS stimulation of feeding as well as JH synthesis. In this view sex peptide initially induces an increase $\mathrm{P}: \mathrm{C}$ intake to levels favorable for anticipated egg production, and the metabolic demand of egg production itself sustains this preference.

An important reason to consider a role for $\mathrm{JH}$ in nutrient preference arises from how $\mathrm{JH}$ affects Drosophila aging. Adult flies lacking JH are longlived [29, 30]. This outcome was reported in both fertile and OvoD sterile adults, suggesting that $\mathrm{JH}$ impacts aging through somatic longevity-assurance physiology rather than as a by-product of reduced egg production. If $\mathrm{JH}$ stimulates high $\mathrm{P}: \mathrm{C}$ intake, $\mathrm{JH}$ knockout flies may be long-lived because they consume low P:C diets. The commonly observed negative correlation between reproductive output and lifespan may occur through parallel somatic and reproductive actions of regulatory hormones, not through allocation of 'resources' to egg production 
at the expense of somatic maintenance [1, 8, 31-33]. One might test this idea by studying the P:C preference of JH knockout Drosophila [30].

We also previously reported that Bam females and males are long-lived [34]. Here we find Bam females mated to fertile wildtype males prefer high P:C diet but based on the results of Lee et al. [1], high P:C consumption is expected to shorten life expectancy. This contradiction might be resolved if Bam-males (as used in Flatt et al. [34]) do not produce sex peptide or do not effectively copulate with Bam females. Further endocrine and demographic studies are needed to resolve these issues.

Our analysis with Drosophila reveals independent, parallel effects of mating hormones and egg production upon P:C dietary intake. We expect these mating related dietary preferences will impact aging. Importantly, how the balance of macronutrients affects invertebrates life history $[1,35,36]$ has been confirmed recently to also occur in mammals. Solon-Biet and colleagues $[37,38]$ studied the impact of protein to carbohydrate (and fat) intake on mouse longevity and reproductive markers. Diets with relatively low $\mathrm{P}: \mathrm{C}$ favored adult survival and age-associated cardiometabolic health while diets with relatively high P:C intake increased steroid-dependent physiological functions positively associated with reproduction in males, and in females as well but to a less consistent extent. The common importance for macronutrient balance among model systems suggest we consider how hormones, reproduction and mating might influence human dietary P:C intake and its associated impacts upon health and aging [39].

\section{Acknowledgments}

Sources of support included NIH R37AG024360 and R01AG031152 to M.T. We extend special thanks to the Tatar lab staff and notably to Dr. Rochele Yamamoto.

\section{References}

[1] Lee KP, Simpson SJ, Clissold FJ, Brooks R, Ballard JW, Taylor PW, et al. Lifespan and reproduction in Drosophila: New insights from nutritional geometry. Proc Natl Acad Sci U S A. 2008;105:2498-503.

[2] Lee KP. Dietary protein: Carbohydrate balance is a critical modulator of lifespan and reproduction in Drosophila melanogaster: A test using a chemically defined diet. J Insect Physiol. 2015;75:12-9.
[3] Skorupa DA, Dervisefendic A, Zwiener J, Pletcher SD. Dietary composition specifies consumption, obesity, and lifespan in Drosophila melanogaster. Aging Cell. 2008;7: 478-90.

[4] Kirkwood TBL, Holliday R. The evolution of ageing and longevity. Proceedings Royal Soceity London Series B. 1979;205:531-46.

[5] Reznick D. Costs of reproduction: An evaluation of the emperical evidence. Oikos. 1985;44:257-67.

[6] Partridge L, Gems D, Withers DJ. Sex and death: What is the connection? Cell. 2005;120(4):461-72.

[7] Westendorp RGJ, Kirkwood TBL. Human longevity at the cost of reproductive success. Nature. 1999;396: 743-6.

[8] O'Brien DM, Min KJ, Larsen T, Tatar M. Use of stable isotopes to examine how dietary restriction extends Drosophila lifespan. Current Biology. 2008;18(4):R155-6.

[9] Chippindale AK, Leroi AM, Kim SB, Rose MR. Phenotypic plasticity and selection in Drosophila life-history evolution. I. Nutrition and the cost of reproduction. J Evol Biol. 1993;6:171-93.

[10] Drummond-Barbosa D, Spradling AC. Stem cell and their progeny respond to nutritinoal changes during Drosophila oogenesis. Developmental Biology. 2001;231:265-78.

[11] Ribeiro C, Dickson BJ. Sex peptide receptor and neuronal TOR/S6K signaling modulate nutrient balancing in Drosophila. Current Biology. 2010;20(11):1000-5.

[12] Vargas MA, Luo N, Yamaguchi A, Kapahi P. A role for S6 kinase and serotonin in postmating dietary switch and balance of nutrients in D. melanogaster. Current Biology. 2010; 20(11):1006-11.

[13] Chapman T, Liddle L, Kalb J, Wolfner M, Partridge L. Male seminal fluid components cause the cost of mating in Drosophila melanogaster females. Nature. 1995;373: 241-4.

[14] Wigby S, Chapman T. Sex peptide causes mating costs in female Drosophila melanogaster. Current Biology. 2005; 15(4):316-21.

[15] Mevel-Ninio M, Terracol R, Kafatos FC. The ovo gene of Drosophila encodes a zinc finger protein required for female germ line development. EMBO Journal. 1991;10: 2259-66.

[16] Ohlstein B, McKearin D. Ectopic expression of the Drosophila Bam protein eliminates oogenic germline stem cells. Development. 1997;124:3651-62.

[17] Kubli E. Sex-peptides: Seminal peptides of the Drosophila male. Cellular and Molecular Life Sciences. 2003;60(8): 1689-704.

[18] Wolfner MF. Tokens of love: Functions and regulation of Drosophila male accessory gland products. Insect Biochem Mol Biol. 1997;27(3):179-92.

[19] Kelly LL. Physiology and biochemistry of the fat body. Advances in Insect Physiology. San Dieago: Academic Press; 1987.

[20] Haunerland NH, Shirk PD. Regional and functional differentiation in the insect fat body. Annual Review of Entomology. 1995;40:121-45.

[21] Bownes M. The regulation of yolk protein gene expression in Drosophila and other flies in higher Diptera. In: Raikhel AS, Sappington TW, editors. Reproductive Biology of Invertebrates. 12. Enfield, USA: Science Publishers; 2004. pp. 95-128. 
[22] Lamnissou K. Nutritional effects on vitellogenesis in species of Drosophila. Journal of Entomological Science. 2000;35: 452-64.

[23] Terashima J, Bownes M. Translating available food into the number of eggs laid by Drosophila melanogaster. Genetics. 2004;167:1711-9.

[24] Rajan A, Perrimon N. Drosophila cytokine unpaired 2 regulates physiological homeostasis by remotely controlling insulin secretion. Cell. 2012;151(1):123-37.

[25] Bai H, Kang P, Tatar M. Drosophila insulin-like peptide6 (dilp6) expression from fat body extends lifespan and represses secretion of Drosophila insulin-like peptide-2 from the brain. Aging Cell. 2012;11(6):978-85.

[26] Junger MA, Rintelen F, Stocker H, Wasserman JD, Vegh M, Radimerski T, et al. The Drosophila forkhead transcription factor FOXO mediates the reduction in cell number associated with reduced insulin signaling. Journal of Biology. 2003;2(3):20.

[27] Puig O, Marr MT, Ruhf ML, Tjian R. Control of cell number by Drosophila FOXO: Downstream and feedback regulation of the insulin receptor pathway. Genes and Development. 2003;17:2006-20.

[28] Soller M, Bownes M, Kubli E. Control of oocyte maturation in sexually mature Drosophila females. Developmental Biology. 1999;208:337-51.

[29] Tatar M, Kopelman A, Epstein D, Tu M-P, Yin C-M, Garofalo RS. A Mutant Drosophila Insulin Receptor Homolog That Extends Life-span and Impairs Neuroendocrine Function. Science. 2001;292:107-10.

[30] Yamamoto R, Bai H, Dolezal AG, Amdam G, Tatar M. Juvenile hormone regulation of Drosophila aging. BMC Biol. 2013;11:85.

[31] Tatar M, Carey JR. Nutrition mediates reproductive tradeoffs with age-specific mortality in the beetle Callosobruchus maculatus. Ecology. 1995;76:2066-73.
[32] Mair W, Sgro CM, Johnson AP, Chapman T, Partridge L. Lifespan extension by dietary restriction in female Drosophila melanogaster is not caused by a reduction in vitellogenesis or ovarian activity. Experimental Gerontology. 2004;39(7):1011-9.

[33] Bell G, Koufopanou V. The cost of reproduction. In: Dawkins R, Ridley M, editors. Oxford Surveys in Evolutionary Biology. 3. Oxford: Oxford University Press; 1986. p. 83-131.

[34] Flatt T, Min KJ, D'Alterio C, Villa-Cuesta E, Cumbers J, Lehmann R, et al. Drosophila germ-line modulation of insulin signaling and lifespan. Proc Natl Acad Sci U S A. 2008;105(17):6368-73.

[35] Maklakov AA, Simpson SJ, Zajitschek F, Hall MD, Dessmann J, Clissold F, et al. Sex-specific fitness effects of nutrient intake on reproduction and lifespan. Current Biology. 2008;18(14):1062-6.

[36] Fanson BG, Weldon CW, Perez-Staples D, Simpson SJ, Taylor PW. Nutrients, not caloric restriction, extend lifespan in Queensland fruit flies (Bactrocera tryoni). Aging Cell. 2009;8(5):514-23.

[37] Solon-Biet SM, Walters KA, Simanainen UK, McMahon AC, Ruohonen K, Ballard JW, et al. Macronutrient balance, reproductive function, and lifespan in aging mice. Proc Natl Acad Sci U S A. 2015;112(11):3481-6.

[38] Solon-Biet SM, McMahon AC, Ballard JW, Ruohonen K, Wu LE, Cogger VC, et al. The ratio of macronutrients, not caloric intake, dictates cardiometabolic health, aging, and longevity in ad libitum-fed mice. Cell Metab. 2014;19(3):418-30.

[39] Le Couteur DG, Solon-Biet S, Cogger VC, Mitchell SJ, Senior A, de Cabo R, et al. The impact of low-protein highcarbohydrate diets on aging and lifespan. Cellular and Molecular Life Sciences. 2016;73(6):1237-52. 